\title{
RESEARCH HIGHLIGHT How cancers usurp macrophages to keep growing
}

\author{
Olmo Sonzogni $\mathbb{D}^{1}$ and Gerburg M. Wulf ${ }^{1}$ \\ Cell Research (2019) 29:423-424; https://doi.org/10.1038/s41422-019-0172-5
}

\begin{abstract}
Liu et al. report the direct interaction of tumor-associated macrophages with cancer stem cells (CSC) through specific ligand-receptor binding (LSECtin-BTN3A3), and how targeting this receptor-ligand interaction decimates the CSC pool.
\end{abstract}

Cancer stem cells (CSC) ${ }^{1}$ have long been viewed as something akin to primordial matter: at times self-starters able to generate an entire tumor when only a few hundred of them are injected, and at other times sleepers that can weather the attack of a chemotherapy treatment only to raise their ugly heads years later.

These persistent CSC have long perplexed researchers: While many of the cellular attributes that maintain the 'stemness' of these populations have been characterized, ${ }^{1}$ we do not know what the signals are that perpetuate their 'stemness' over time or awaken them after a long dormancy period.

The work by Liu et al. ${ }^{2}$ provides novel understanding towards that riddle: From a series of carefully conducted experiments they conclude that the maintenance of 'stemness' is actually not the result of cell-autonomous mechanisms, but cells in the tumor microenvironment, specifically tumor-associated macrophages (TAMs), decisively contribute to securing a pool of CSC for a tumor. They identify a signaling axis that requires direct TAMcancer cell interaction mediated by LSECtin on TAMs and its receptor BTN3A3 on tumor cells. BTN3A proteins belong to the B7 family of transmembrane type II Immunoglobulins and all three members of the BTN3A subfamily, ${ }^{3}$ BTN3A1, BTN3A2 and BTN3A3, are expressed in various types of cancer cells. ${ }^{4}$ Recent reports associate single nucleotide variations in BTN3A2 and BTN3A3 with increased susceptibility to gastric and ovarian cancers, ${ }^{5}$ suggesting a likely, but not yet well-defined involvement of these transmembrane proteins in cancer growth and progression.

Termed a pathogen receptor, LSECtin is a single-pass, type II transmembrane glycoprotein known to bind to mannose, $\mathrm{N}$-Acetylglucosamine, and fucose. Through binding to the surface glycoproteins of enveloped viruses, LSECtin mediates the uptake of these viruses. ${ }^{6,7}$ Interestingly, in this work Liu et al. show that LSECtin effectively mediates tolerance of CSCs. They start off with the observations that expression of LSECtin on macrophages is generally induced by IL-4 and IL-13. Intriguingly, they find that co-culture with tumor cells will also induce LSECtin expression on tumor-associated myeloid cells, and mostly on TAMs. Using macrophages with LSECtin extinction and those with LSECtin overexpression, they demonstrate that LSECtin-expressing macrophages greatly enhance the efficiency of tumor initiation and tumor growth in models of triple-negative breast cancer. As direct physical interaction was necessary for LSECtin-expressing macrophages to maintain CSC, the authors then proceeded to clone the LSECtin receptor by using HEK293 cells that did not bind to LSECtin and therefore lacked the putative receptor. The authors transfected these HEK293 cells with a cDNA library and screened LSECtin-binding cells, identifying BTN3A3 as the high-affinity binding partner for LSECtin. Underscoring the potential relevance of the LSECtin-BTN3A3 interaction in human breast cancer, they found both LSECtin-overexpressing macrophages and BTN3A3-overexpressing tumor cells in clinical breast cancer specimen. Consistent with a pro-CSC activity, they observed higher levels of BTN3A3 in ER-negative breast cancers, and a trend of poor outcomes in those tumors that were BTN3A3 high in the ER-positive subset of breast cancer. In tumor cells, BTN3A3 occupation by a ligand, LSECtin or an agonist anti-BTN3A3 antibody, activated the JAK-STAT pathway, indicating that maintaining a CSC pool is an active and energy-consuming process.

Amongst the anti-BTN3A3 antibodies that they generated, they found both blocking (5E08) and activating (31H03) antibodies that could slow (5E08) or accelerate $(31 \mathrm{H03})$ the growth of CSC. The blocking 5E08 antibody, when given in vivo, greatly enhanced the efficacy of Paclitaxel, suggesting that co-targeting the chemotherapy-resistant stem cell pool as well as proliferating cancer cells is potentially a strategy to improve treatment outcomes.

The work by Liu et al. shows that tumors actively curate a CSC pool by recruiting TAMs, hence ensuring survival and regrowth of the tumor if it is decimated but not eliminated by chemotherapy, radiation or surgery. Evolutionarily, this mechanism might have served constructive purposes in the non-malignant setting, e.g., when an influx of M2 macrophages might facilitate wound healing by promoting the maintenance and expansion of a pool of pluripotent epithelial cells with high proliferative potential. Epithelial tumors such as breast cancer would thus have maintained this mechanism to curate a pool of CSC, to add to its growth advantage.

However, this dependence on the TAM-CSC interaction may also be a vulnerability as Liu et al. show. Clearing $M 2$ macrophages in a tumor has anti-tumor effects, not only immediately, but also long term by abolishing CSC that might otherwise give rise to late relapses. The combination of a taxane with blocking anti-BTN3A3 antibodies is a fine example of efforts to co-target tumor cells and their supporting microenvironment. It could potentially be translated into a clinical trial if and when anti-BTN3A3 antibodies are available for human study and have cleared first-in human studies. These treatments might not only be beneficial because of potentially non-overlapping toxicities, but also non-overlapping targets: while chemotherapy such as taxanes would decimate the cycling tumor cells acutely, blocking BTN3A3 on TAMs might improve long-term outcomes by decimating the CSC population, and decrease the risk of relapse. Notably, the lessons here might not only apply to the potential use of anti-BTN3A3

${ }^{1}$ Beth Israel Deaconess Medical Center, Department of Medicine, Harvard Medical School, Boston, MA 02215, USA

Correspondence: Gerburg M. Wulf (gwulf@bidmc.harvard.edu)

Published online: 6 May 2019 
424

antibodies, but also to the therapeutic use of anti-CSF1R antibodies, HDAC inhibitors or PI3KY inhibitors that have already advanced to clinical trials. ${ }^{8-}$

Lastly, the LSECtin/BTN3A3 axis illustrates that tumors maintain a degree of organismal structure that can be targeted. Investing in a CSC pool is an energy-consuming process that requires continuous signaling through the JAK/STAT pathway, a potentially second nodal point for intervention. The requirement for a direct cell-cell contact ensures that this privilege is bestowed only on a select few cancer cells, part of a protective program that TAMs offer cancer cells in their immediate vicinity. Clearly, this work kick-starts further investigation: Does this concept apply also to ER-positive breast cancer where patients suffer relapses often after decades? Does it apply to solid tumors in general? Besides LSECtin expression, what are the characteristics of the TAMs that support CSC? And what role do these TAMs play in maintaining the plasticity of cancer cells, i.e., can these TAMs aide in the generation of CSC from ordinary cancer cells?

\section{REFERENCES}

1. Batlle, E. \& Clevers, H. Nat. Med. 23, 1124-1134 (2017).

2. Liu, D. et al. Cell Res. 29, 365-378 (2019).

3. Henry, J. et al. Immunol. Today. 20, 285-288 (1999).

4. Cubillos-Ruiz, J. R. et al. Oncotarget 1, 329-338 (2010).

5. Peedicayil, A. et al. PLoS ONE 5, e8884 (2010).

6. Powlesland, A. S. et al. J. Biol. Chem. 283, 593-602 (2008).

7. Dominguez-Soto, A. et al. Blood 109, 5337-5345 (2007).

8. Pathria, P., Louis, T. L. \& Varner, J. A. Trends Immunol. 40, 310-327 (2019).

9. Guerriero, J. L. et al. Nature 543, 428-432 (2017).

10. Quail, D. F. et al. Science 352, aad3018 (2016). 